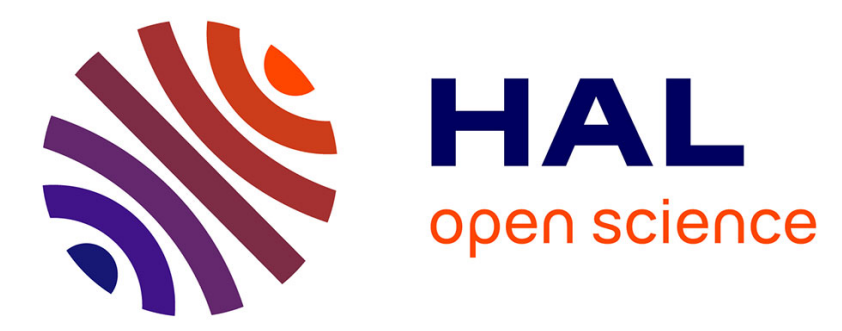

\title{
A quantum delayed-choice experiment enabled by entanglement
}

Florian Kaiser, Thomas Coudreau, Daniel Barry Ostrowsky, Pérola Milman, Sébastien Tanzilli

\section{- To cite this version:}

Florian Kaiser, Thomas Coudreau, Daniel Barry Ostrowsky, Pérola Milman, Sébastien Tanzilli. A quantum delayed-choice experiment enabled by entanglement. 22nd International Laser Physics Workshop (LPHYS'13), Jul 2013, Prague, Czech Republic. hal-00847629

\section{HAL Id: hal-00847629 \\ https://hal.science/hal-00847629}

Submitted on 24 Jul 2013

HAL is a multi-disciplinary open access archive for the deposit and dissemination of scientific research documents, whether they are published or not. The documents may come from teaching and research institutions in France or abroad, or from public or private research centers.
L'archive ouverte pluridisciplinaire $\mathbf{H A L}$, est destinée au dépôt et à la diffusion de documents scientifiques de niveau recherche, publiés ou non, émanant des établissements d'enseignement et de recherche français ou étrangers, des laboratoires publics ou privés. 


\title{
A quantum delayed-choice experiment enabled by entanglement
}

\author{
Florian Kaiser ${ }^{1}$, Thomas Coudreau ${ }^{2}$, Perola Milman ${ }^{2}$, Daniel B. Ostrowsky ${ }^{1}$, Sébastien Tanzilli ${ }^{1}$ \\ ${ }^{1}$ LPMC, Université Nice - Sophia Antipolis, CNRS UMR 7336, Parc Valrose, 06108 Nice, France \\ ${ }^{2}$ Laboratoire MPQ, Université Paris Diderot, Sorbonne Paris Cité, CNRS UMR 7162, 75013 Paris, France
}

Bohr's complementarity notion is at the heart of quantum physics. It suggests that quantum systems are observed as waves or particles depending on the type of measurement, i.e. the experimental arrangement, they are subjected to [1]. For instance (see FIG. 1), sending single photons to an open or closed Mach-Zehnder interferometer (MZI) leads to the observation of particle or wave behaviours, respectively [2]. In 1984, Wheeler proposed a "Gedanken" delayed-choice experiment in which the interferometer configuration is chosen at will by the experimentalist only after the photon has already passed the input beam-splitter $\left(\mathrm{BS}_{\mathrm{in}}\right)$ of the device [3]. This experiment was realized using a single photon source and showed that Bohr's complementarity notion was still obeyed [4].

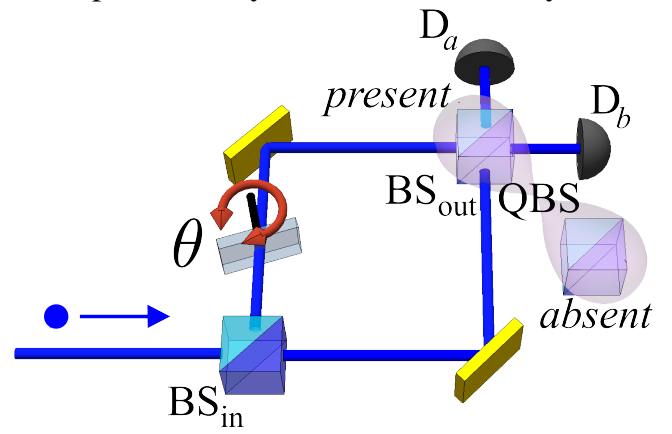

FIG. 1. Setup for observing single photon wave-particle complementarity based on a MZI. When the output beam-splitter $\left(\mathrm{BS}_{\text {out }}\right)$ is absent, a click on detector $\mathrm{D}_{a}$ or $\mathrm{D}_{b}$ reveals a particle behaviour. When $\mathrm{BS}_{\text {out }}$ is present, phase $(\theta)$ dependent intensity oscillations are observed in these detectors, revealing a wave phenomenon $[3,4]$. In our quantum version of Wheeler's experiment, $\mathrm{BS}_{\text {out }}$ is prepared in a coherent superposition of being present and absent by exploiting entanglement, leading to the implementation of a quantum beam-splitter (QBS) $[5,6]$.

It was recently proposed to take Wheeler's experiment one step further by employing an output "quantum beamsplitter" (QBS), i.e. preparing $\mathrm{BS}_{\text {out }}$ in a coherent superposition of being absent and present [5]. This allows choosing the type of measurement, i.e. wave or particle, only after having determined the state of the QBS, which can be (in principle) infinitely delayed. We realized such an experiment by exploiting the resource of entanglement, in this case pairs of polarization entangled photons. The behaviour of one of the paired photons, called the test photon, is analysed using a MZI having a QBS at its output. This QBS is enabled by entanglement and made of a po- larization dependent beam-splitter (not shown). The other photon, called the corroborative photon, allows determining the state of the QBS, and consequently, which test photon behaviour (wave, particle, or both) is observed.

By manipulating the corroborative photon polarization state, we demonstrate a continuous morphing of the test photon from wave to particle behaviour. This refutes simple models of single photons behaving exclusively as waves or particles (see FIG. 2) [6]. The state of the QBS is determined via the measurement of the corroborative photon (not shown) only after the test photon has already been detected. The space-like separation between the two measurements invalidates local-hidden variable models associated with pre-existing information about the measurement outcomes. In other words, when the test photon is detected, no information is available about the type of measurement it underwent. These results still perfectly obey Bohr's complementarity notion and its extension [7].

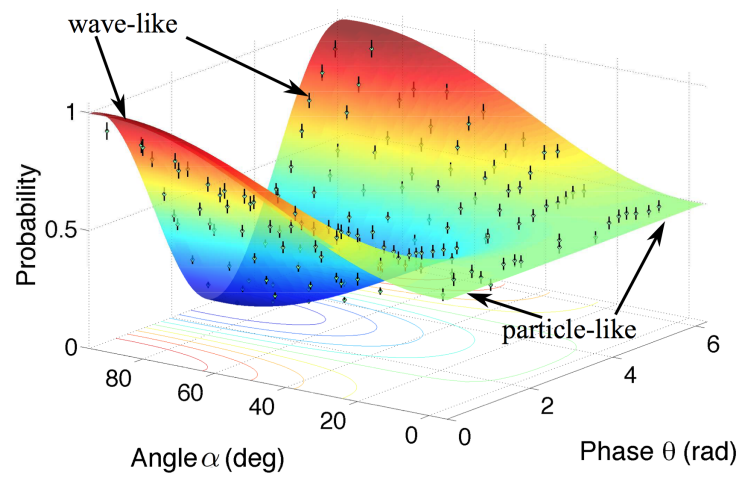

FIG. 2. Continuous morphing from wave to particle-like behaviours when manipulating the QBS at the output of our MZI (see FIG. 1). $\theta$ is a tunable phase in one arm of the interferometer, while $\alpha$ is the polarization state anlysis angle of the corroborative photon.

Entanglement is at the heart of our approach. It permits observing genuine quantum behaviour for the test photons. The entanglement quality is verified by the violation of the Bell inequalities with more than 10 standard deviations [6].

Trying to explain the results of our quantum version of Wheeler's experiment in classical terms causes severe contradictions, but the results are in perfect agreement with quantum physics, in which the measurement timing order does not matter. The beauty of such an experiment is that space and time do not seem to play any role $[6,8,9]$.
[1] N. Bohr, Nature 121, 580-590 (1928).

[2] P. Grangier et al., Europhys. Lett. 1, 173-179 (1986).

[3] N. Bohr in Quantum theory and measurement, J. A. Wheeler, W. H. Zurek, Eds. (Princeton Univ. Press), p. 949 (1984).

[4] V. Jacques et al., Science 315, 966-968 (2007).
[5] R. Ionicioiu et al., Phys. Rev. Lett. 107, 230406 (2011).

[6] F. Kaiser et al., Science 338, 637-640 (2012).

[7] B.-G. Englert, Phys. Rev. Lett. 77, 2154-2157 (1996).

[8] A. Peruzzo et al., Science 338, 634-637 (2012).

[9] S. Lloyd, Science 338, 621-622 (2012). 